\title{
Synthesis of Neopentyl Biphenylsulfonates Using the Suzuki-Miyaura Reaction
}

\author{
Chul-Hec Cho, Chul-Bac Kìm, Myungchul Sun, and Kwangyong Park’
}

\author{
Deparment of Chemical Engineering, Chung-Ang University, Seotl 156-756. Korea \\ Received Angust 21, 2003
}

\begin{abstract}
Palladium-catalyzed cross-coupling reactions of neopentyloxysulfonylphenyl bromides with arylboronic acids provided a variety of neopentyl biphenylsulfonates in good yields. 2-Bromo- and 4-bromobenzenesulfonates underwent the coupling reaction more rapidly than 3-bromobenzenesulfonate, while chlorobenzenesulfonate did not produce the coupling product under the standard reaction conditions.
\end{abstract}

Key Words: Cross-coupling, Bromobenzenesulfonates, Biphenylsulfonales, Arylboronic acid, SuzukiMiyaura reaction

\section{Introduction}

Transition metal-catalyzed coupling reaction is one of the most important synthetic tools for constructing carboncarbon bonds.' In particular, nickel- and palladium-catalyzed coupling reactions of organic halides and pseudohalides with organoboronic acids. ${ }^{2}$ organostannanes, ${ }^{3}$ organozines, ${ }^{+}$and Grignard reagents' have energed as extremely powerlul methods during the last several decades. Among those processes, the Suzuki-Miyaura reaction of aryl and vinyl halides/triflates with organoboronic acids is prelerted in many situations. This popularity can be atributed to the stability and low toxicity of boronic acids as well as the mild reaction conditions.

The preparation of asymmetric biaryls, which are of great interest due to their biological ${ }^{6}$ and optical properties, has been the object of transition metal-catalyzed coupling processes. Since the preparation of biaryls via the palladiumcatalyzed reaction of arylboronic acids with aryl halides was lirst reported. ${ }^{8}$ this reaction has been used successlully for a variety of aryl halides and triflates. Recently, it has become widely applied in solid-phase organic synthesis (SPOS) for the rapid and convenient preparation of asymmetric biaryl libraries." However, the coupling reaction of aryl electrophiles containing sulfur-substituents, such as thio, sulfinyl. and sulfonyl groups, with organoboronic acids has not been explored except for the reactions of $p-\mathrm{BrC}_{6} \mathrm{H}_{4} \mathrm{SO}_{3} \mathrm{Na}^{10}$ and bromobenzenesulfonamide. "l

As part of an ongoing study directed toward the development of cross-coupling reactions between alkyloxysulfonylarenes and aryl nucleophiles, ${ }^{12}$ a variety of neopentyl biphenylsulfonates were required. They could be produced by the palladiumcatalyzed coupling reactions of alkyl bromobemzenesulfonates with aryl boronic acids in good yields. To the best of our knowledge, this is the first general case of a cross-coupling reaction of sulfur-substituted aryl electrophiles with organoboronic acids. This paper reports our efforts in preparing neopentyl biphenylsulfonates via the Suzuki-Miyaura reaction.

\footnotetext{
"To whom correspondence should be addressed. e-mail: kypark (a)cau.ac.kr
}

\section{Results and Discussion}

The alkyl halobenzenesulfonales I were prepared using a previously reported procedure (Scheme 1). ${ }^{12}$ Two types of neopentyl moicties, 2,2-dimelhyl-3-phenyl-1-propyl and 2,2-dimethyl-1-propyl, were selected as the alkyl groups for these intermediates. All the sulfonates 1 were produced in good yields (Table 1). Products 1a, 1b, and ld were purilied by recrystallization from $n$-hexane to give whic solids, while le was isolated by column chromalography $\left(\mathrm{El}_{2} \mathrm{O}: n-\right.$ hexane - 1:4) to allord a clcar oil.

The palladium-catalyzed cross-coupling reaction of I with arylboronic acids proceeded smoothly in the presence of

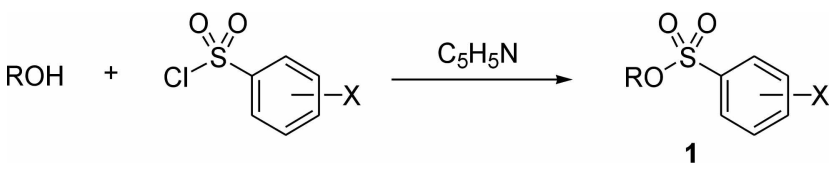

Scheme 1

Table 1. Preparation of Neopentyl halobsnzenesulfonates $1^{a}$

\begin{tabular}{|c|c|c|c|c|}
\hline Entry & $\mathrm{R}^{\prime \prime}$ & $X$ & $\begin{array}{c}\text { Sulfonate } \\
1\end{array}$ & $\begin{array}{l}\text { Yield } \\
(\%))^{\prime}\end{array}$ \\
\hline I & $\mathrm{R}^{\prime}$ & $4-\mathrm{Br}$ & & 87 \\
\hline 2 & $\mathrm{R}^{2}$ & $3-\mathrm{Br}$ & & 81 \\
\hline 3 & $\mathrm{R}^{2}$ & $2-\mathrm{Hr}$ & & 68 \\
\hline 4 & $\mathrm{R}^{2}$ & 4-Cl & ${ }_{1 d}$ & 87 \\
\hline
\end{tabular}

"Reactions of the alcohols $(51.7 \mathrm{mmol})$ with sulfomy chlorides $(47.0$ momol) were carried out in chloroform $(50 \mathrm{mI}$ ) in the presence of pyridine $(103.0 \mathrm{mmol}) .{ }^{2} \mathrm{R}^{1}=2.2$-dimethyl-3-phenyl-I-propyl. $\mathrm{R}^{2}=$ neopentyl. 'lsolated yields based on sulionyl chlorides. 


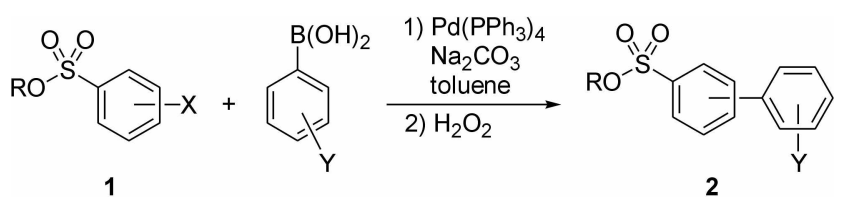

Sclieme 2

Table 2. Coupling Ractions of $\mathbf{I}$ with ary lboronic acidst

\begin{tabular}{|c|c|c|c|c|c|}
\hline Inntry & 1 & $\begin{array}{l}\text { Boronic } \\
\text { acid (Y) }\end{array}$ & $\begin{array}{l}\text { Time } \\
\text { (h) }\end{array}$ & $\begin{array}{c}\text { Producl } \\
2^{h}\end{array}$ & $\begin{array}{l}\text { Yicld } \\
(\%)^{\prime}\end{array}$ \\
\hline 1 & la & II & 6 & $2 a$ & 71 \\
\hline 2 & la & 4-'Bu & 6 & 2b & 78 \\
\hline 3 & Ia & 4-vityl & 6 & & 76 \\
\hline 4 & Ia & 4-Ph & 6 & $2 d$ & 68 \\
\hline 5 & 1a & 4-CHIO & 6 & $2 e$ & 64 \\
\hline 6 & & $\mathrm{HO}-4-(\mathrm{OCll}$ & 6 & $2 f$ & 374 \\
\hline 7 & $1 b$ & 4-CIIO & 15 & & 69 \\
\hline 8 & lc & $4-\mathrm{CHO}$ & 6 & $2 \mathrm{~h}$ & 66 \\
\hline 9 & ld & $4-\mathrm{Cl}_{3}$ & 15 & $2 \mathbf{i}$ & - \\
\hline 10 & 1d & 4-'Bu & 15 & & - \\
\hline
\end{tabular}

"Reactions of 1 (5.22 mmol) with ary lboronic acids $(5.74 \mathrm{mmol})$ in the presence of $\mathrm{Pd}(\mathrm{PPh})_{+}(0.157 \mathrm{mmol})$ and acueous sodium carbonats $(2.0$ M. $6.0 \mathrm{~mL})$ were carried out in the refluxing temperature of toluene $(12.0$ $\mathrm{mL}$ ) under at $\mathrm{Ar}$ atmosphere. "R' 2.2-dimetlyl-3-phenyl-1-propyl. $R$ " $=$ neopenty. "Isolated tickds based on 1 .

sodium carbonate (Scheme 2). Although a detailed study to optimize the reaction conditions was not undertaken, most of the reactions were complete within $6 \mathrm{~h}$ at the refluxing temperature of toluene. 'The displacement of arenesulfonates ${ }^{1.3}$ or alkyloxysulfonyl groups ${ }^{12}$ was not observed under the standard reaction conditions.
The result of the cross-coupling reactions between $\mathbf{1}$ and the various arylboronic acids is summarized in Table 2. 4Bromobenzenesulfonate Ia reacted rapidly with phenyl-, 4tert-butylphenyl-, 4-vinylphenyl-, and biphenylboronic acids in the presence of $\mathrm{Pd}\left(\mathrm{P}^{\mathrm{P}} \mathrm{h}_{3}\right)_{4}$ catalyst to give the corresponding biphenyl- and terphenylsulfonates 2 in good yields (entries 1-4). While formylphenylboronic acid, which has an electronwithdrawing substituent, showed a slightly lower reactivity than the above nucleophiles (entry 5), 3-formyl-4-methoxyphenylboronic acid underwent the coupling reaction rapidly (entry 6).

The reaction of 3-bromobenzenesulfonate lb with 4 formylphenylboronic acid also produced the desired coupling product in good yield, although it required more time ( $15 \mathrm{~h}$ ) for a complete reaction (entry 7). 2-Bromobenzenesulfonate 1c underwent the coupling process with formyl-phenylboronic acid in a similar way as with $\mathbf{1 a}$ (entry 8 ). There was no evidence showing that the neighboring neopentyloxysulfonyl group provided a significant steric hindrance for the coupling reaction.

Chlorobenzenesulfonate ld was quite inert under our standard reaction conditions. Even though it is well known that chlorobenzene derivatives are generally not reactive toward the oxidative addition of a palladium catalyst, some electron-deficient aryl chlorides have been reported to undergo the palladium-catalyzed coupling reaction with organoboronic acids successfully. ${ }^{14}$ However, reactions of $1 \mathbf{d}$ with 4-methylphenyl- and 4-tert-butylphenylboronic acids did not give any noticeable amounts of the desired coupling products in this effort (entries 9 and 10 ).

\section{Conclusion}

In conclusion, the palladium-catalyzed cross-coupling reaction of neopentyloxysulfonylphenyl bromides with arylboronic acids in the presence of base was described. A variety of neopentyl biphenylsulfonates were prepared in good yields. To our knowledge, this is the first general exploration of the Suzuki-Miyaura reaction of sulfursubstituted aryl electrophiles. Investigations into applying this coupling strategy for preparing asymmetric terphenyl and stilbene derivatives are currently underway and will be reported in due course.

\section{Experimental Section}

${ }^{\mathrm{J}} \mathrm{H}$ NMR (300 or $500 \mathrm{MHz}$ ) and ${ }^{13} \mathrm{C}$ NMR (75 or $125 \mathrm{MHz}$ ) were registered in $\mathrm{CDCl}$ s as solvent and tetramethylsilane (IMS) as internal standard. Chemical shifts are reported in $\delta$ units (ppm) by assigning ' IMS resonance in the ${ }^{1} \mathrm{H}$ spectrum as $0.00 \mathrm{ppm}$ and $\mathrm{CDCls}$ resonance in the ${ }^{13} \mathrm{C}$ spectrum as 77.2 ppm. All coupling constants, $J$, are reported in hertz $(\mathrm{Hz})$. Column chromatography was performed on silica gel 60,70 230 mesh. Analytical thin-layer chromatography (ILC) was performed using Merck Kieselgel $60 \mathrm{~F}_{254}$ precoated plates $(0.25 \mathrm{~mm})$ with a fluorescent indicator and visualized with UV light (254 and $365 \mathrm{~nm}$ ) or by iodine vapor staining. GC 
analysis was performed on a bonded $5 \%$ pheny lpolysiloxane BPX 5 capillary column ( $\mathrm{SGE}, 30 \mathrm{~m}, 0.32 \mathrm{mum}$ i.d.). Electron impact (EI. $70 \mathrm{eV}$ ) was used as the ionization method for the mass spectrometry. Melting points were obtained using a Barnstead/Thermolyne MEL-TEMP apparatus and are uncorrected. Toluene was distilled from calcium hydride. 4-Tolyl- 4-tert-butylphenyl- 4-vinylphenyl- and biphenylboronic acid were prepared according to a literature procedure ${ }^{\text {s }}$ Phenyl- 4-formylphenyl-, and 3-fonnyl-4methoxyphenylboronic acid were used as obtained commercially

General Procedure for the Preparation of Neopentyl Halobenzenesulfonates (1). To the solution of alcohol (51.7 mmol) in chloroform $(50 \mathrm{~mL})$ at $0{ }^{\circ} \mathrm{C}$ were added pyridine (103.0 mmol) dropwise over a period of $20 \mathrm{~min}$ and halobenzenesulfonyl chloride $(47.0 \mathrm{mmol})$ in small portions. The reaction mixture was stirred at room temperature for 12 $\mathrm{h}$ and diluted with $\mathrm{Et} \mathrm{O}_{2} \mathrm{O}$. The solution was washed with a $0.1 \%$ aqueous $\mathrm{HCl}$, water, and brine: dried over $\mathrm{MgSO}_{4}$ : filtered: and concentrated in vacuo.

2,2-Dimethyl-3-phenyl-1-propyl $\quad 4^{\prime}$-bromobenzenesulfonate (1a) was prepared by the reaction of 2.2-dimethyl3-phenyl-1-propanol (8.48 g. $51.7 \mathrm{mmnol}$ ) with p-bronobenzenesulfonyl chloride $(12.0 \mathrm{~g} .47 .0 \mathrm{mmol})$. The crude compound was purified by recrystallization from $n$-hexane to give $1 \mathrm{a}(15.66 \mathrm{~g} .87 \%)$ as a white solid: TLC $R_{f} 0.38$ $\left(\mathrm{Et}_{2} \mathrm{O}: n\right.$-hexane $\left.=\mathrm{l}: 4\right) ; \mathrm{mp} 82.83{ }^{\circ} \mathrm{C}:{ }^{1} \mathrm{H}$ NMR $(300 \mathrm{MHz}$. $\left.\mathrm{CDCl}_{3}\right) \delta 0.88(\mathrm{~s}, 6 \mathrm{H}), 2.55$ (s. $\left.2 \mathrm{H}\right) .3 .68(\mathrm{~s}, 2 \mathrm{H}), 6.99-7.03$ (m, $2 \mathrm{H}) .7 .19-7.22(\mathrm{~m}, 3 \mathrm{H}) .7 .71(\mathrm{~d}, J=8.73 \mathrm{~Hz}, 2 \mathrm{H}) .7 .79$ (d, $J=8.73 \mathrm{~Hz}, 2 \mathrm{H}):{ }^{13} \mathrm{C}$ NMR $\left(75 \mathrm{MHz}, \mathrm{CDCl}_{3}\right) \delta 24.1$ $(\times 2), 35.4,44.3,77.7,126.6,128.2(\times 2), 129.2,129.7(\times 2)$. $130.6(\times 2), 132.9(\times 2), 135.3,137.5$; HRMS (EI. $70 \mathrm{eV})$ calcd for $\mathrm{C}_{17} \mathrm{H}_{19} \mathrm{BrO}_{3} \mathrm{~S}\left(\mathrm{M}^{-}\right) 382.0238$. found 382.0231 .

Neopentyl 3-bromobenzenesulfonate (1b) was prepared by the reaction of neopentyl alcohol $(0.36 \mathrm{~g} .4 .09 \mathrm{~mm}$ mol) with $m$-bromobenzenesulfonyl chloride $(0.95 \mathrm{~g} .3 .72 \mathrm{mmol})$. The crude compound was purified by recrystallization from $n$ hexane to give $1 \mathrm{~b}(0.93 \mathrm{~g} .81 \%)$ as a white solid: TLC $R_{f}$ $0.51\left(\mathrm{Et}_{2} \mathrm{O}: n\right.$-hexane $\left.=1: 1\right) ; \mathrm{mp} 44-45^{\circ} \mathrm{C}:{ }^{1} \mathrm{H}$ NMR $(300$ $\left.\mathrm{MHz}, \mathrm{CDCl}_{\mathrm{s}}\right) \delta 0.92(\mathrm{~s}, 9 \mathrm{H}), 3.72(\mathrm{~s}, 2 \mathrm{H}), 7.45$ (dd. $J=8.06$. $7.89 \mathrm{~Hz} .1 \mathrm{H}) .7 .79(\mathrm{ddd} . J=8.06 .2 .01 .1 .00 \mathrm{~Hz} . \mathrm{lH}) .7 .85$ (ddd $J=7.89,1.68,1.00 \mathrm{~Hz}, 1 \mathrm{H}), 8.06(\mathrm{dd}, J=2.01,1.68$ $\mathrm{Hz}, 1 \mathrm{H}):{ }^{13} \mathrm{C}$ NMR $\left(125 \mathrm{MHz} . \mathrm{CDCl}_{3}\right) \delta 26.2(\times 3), 31.9$. $80.3,123.3,126.5,130.9,130.9,136.9,138.1$ : HRMS (EI. $70 \mathrm{eV}$ ) calcd for $\mathrm{C}_{11} \mathrm{H}_{1:} \mathrm{BrO}_{3} \mathrm{~S}\left(\mathrm{M}^{-}\right) 305.9925$. found 305.9969 .

Neopentyl 2-bromobenzenesulfonate (1c) was prepared by the reaction of neopentyl alcohol $(0.57 \mathrm{~g} .6 .46 \mathrm{mmol})$ with $o$-bromobenzenesulfonyl chloride $(1.50 \mathrm{~g} .5 .87 \mathrm{mmol})$. The crude compound was purified by column chromatography $\left(\mathrm{Et}_{z} \mathrm{O}: n\right.$-hexane $\left.=1: 4\right)$ to afford $1 \mathrm{c}(1.23 \mathrm{~g} .68 \%)$ as a viscous colorless oil: $\mathrm{TLC} R_{f} 0.56\left(\mathrm{Et}_{2} \mathrm{O}: n\right.$-hexane $=$ l: 1): ${ }^{1} \mathrm{H}$ NMR (300 MHz. CDCl $) \delta 0.96$ (s. 9H), 3.73 (s. 2H). 7.48-7.55 (m. 2H). 7.77-7.82 (m. 1H) 8.09-8.14 (m. 1H): ${ }^{13} \mathrm{C}$ NMR $\left(75 \mathrm{MHz}, \mathrm{CDCl}_{3}\right) \delta 26.0(\times 3), 31.5,80.4$. 120.6. 127.8. 132.0. 132.2, 134.8, 135.7: HRMS (EI. $70 \mathrm{eV}$ ) calcd for $\mathrm{C}_{11} \mathrm{H}_{1:} \mathrm{BrO}_{3} \mathrm{~S}\left(\mathrm{M}^{-}\right) 305.9925$. found 305.9948 .

Neopentyl 4-chlorobenzenesulfonate (1d) was prepared by the reaction of neopentyl alcohol $(1.84 \mathrm{~g} .20 .85 \mathrm{mmol})$ with $p$-chlorobenzenesulfonyl chloride $(4.00 \mathrm{~g} .18 .95 \mathrm{mmol})$. The crude compound was purified by recrystallization from $n$ hexane to give $1 \mathrm{~d}(4.33 \mathrm{~g} .87 \%)$ as a white solid: TLC $R_{f}$ $0.63\left(\mathrm{Et}_{2} \mathrm{O}: n\right.$-hexane $\left.=1: 1\right): \mathrm{mp} 64{ }^{\circ} \mathrm{C}:{ }^{1} \mathrm{H}$ NMR $(300$ $\left.\mathrm{MHz}, \mathrm{CDCl}_{3}\right) \delta 0.91$ (s, 9H). 3.70 (s. $2 \mathrm{H}$ ), 7.54 (d. $J=8.56$ Hz. $2 \mathrm{H}$ ). 7.85 (d. $J=8.56 \mathrm{~Hz}, 2 \mathrm{H}$ ): ${ }^{13} \mathrm{C}$ NMR $(75 \mathrm{MHz}$, $\left.\mathrm{CDCl}_{3}\right) \delta 26.0(\times 3), 31.7,80.1,129.5(\times 2) .129 .8(\times 2)$, 134.8, 140.5; HRMS (EI, $70 \mathrm{eV}$ ) calcd for $\mathrm{C}_{11} \mathrm{H}_{15} \mathrm{ClO}_{3} \mathrm{~S}$ (M) 262.0430, found 262.0434

General Procedure for the Coupling Reaction of 1 with arylboronic acid. To the solution of halobenzenesulfonate $(5.22 \mathrm{mmol})$ and $\mathrm{Pd}\left(\mathrm{PPl}_{3}\right)_{4}(0.157 \mathrm{mmol})$ in toluene $(12$ $\mathrm{mL}$ ) was added $2.0 \mathrm{M}$ aqueous $\mathrm{Na}_{2} \mathrm{CO}_{3}(6.0 \mathrm{~mL})$ under an Ar atmosphere. To the resulting mixture was added arylboronic acid $(5.74 \mathrm{mmol})$. which was dissolved in ethanol $(3 \mathrm{~mL})$. The reaction misture was heated at reflux for $6 \mathrm{~h}$ with vigorous stirring. To the resulting mixture was added $30 \%$ hydrogen peroxide $(0.3 \mathrm{~mL})$ to oxidize the residual boronic acid. The mixture was stirred at room temperature for $\mathrm{l} \mathrm{h}$ and diluted with EtOAc. The organic layer was washed with water and brine; dried over $\mathrm{MgSO}_{4}$; filtered through a small pad of silica gel in a sintered glass filter; and concentrated in vacuo.

2,2-Dimethyl-3-phenyl-1-propyl 1',1"-biphenyl-4'-sulfonate (2a) was prepared by the reaction of $1 \mathrm{a}(2.00 \mathrm{~g} .5 .22$ mumol) with phenylboronic acid (0.70 g. $5.74 \mathrm{mmol})$ in the presence of $\mathrm{Pd}\left(\mathrm{PPh}_{3}\right)_{4}(0.181 \mathrm{~g}, 0.157 \mathrm{mmol})$ and $2 \mathrm{M}$ aq. $\mathrm{Na}_{2} \mathrm{CO}_{3}(6.0 \mathrm{~mL})$ by using toluene $(12.0 \mathrm{~mL})$ as solvent. The crude product was purified by recrystallization from $n$ hexane to give $2 \mathrm{a}(1.4 \mathrm{~g} .71 \%)$ as a white solid: TLC $R_{f}$ $0.43\left(\mathrm{Et}_{2} \mathrm{O}: n\right.$-hexane $\left.=1: 4\right): \mathrm{mp} 74.75^{\circ} \mathrm{C} ;{ }^{1} \mathrm{H}$ NMR $(300$ $\left.\mathrm{MHz}, \mathrm{CDCl}_{3}\right) \delta 0.89(\mathrm{~s}, 6 \mathrm{H}), 2.57(\mathrm{~s}, 2 \mathrm{H}), 3.72(\mathrm{~s}, 2 \mathrm{H}), 7.00-$ 7.05 (m. $2 \mathrm{H}), 7.15-7.2 \mathrm{l}(\mathrm{m}, 3 \mathrm{H}) .7 .42-7.53(\mathrm{~m} .3 \mathrm{H}), 7.63(\mathrm{~d}$. $J=6.72 \mathrm{~Hz} .2 \mathrm{H}), 7.76($ d. $J=8.73 \mathrm{~Hz}, 2 \mathrm{H}), 7.99($ d. $J=8.73$ $\mathrm{Hz}, 2 \mathrm{H}) ;{ }^{13} \mathrm{C} \mathrm{NMR}\left(75 \mathrm{MHz} . \mathrm{CDCl}_{3}\right) \delta 24 . \mathrm{l}(\times 2), 35.4,44.3$, $77.4,126.5 .127 .6(\times 2), 128.1(\times 2), 128.2(\times 2) .128 .7(\times 2)$, $129.0 .129 .4(\times 2), 130.7(\times 2), 134.7 .137 .6 .139 .3,147.0$. HRMS (EI. $70 \mathrm{eV}$ ) calcd for $\mathrm{C}_{23} \mathrm{H}_{24} \mathrm{O}_{3} \mathrm{~S}\left(\mathrm{M}^{-}\right) 380.1446$, found 380.1403 .

2,2-Dimethyl-3-phenyl-1-propyl +"-tert-butyl-1',1"biphenyl-t'-sulfonate (2b) was prepared by the reaction of $1 \mathrm{a}(2.00 \mathrm{~g} .5 .22 \mathrm{mmol})$ with $p$-tert-butylphenylboronic acid $(1.02 \mathrm{~g} .5 .74 \mathrm{mmol})$ in the presence of $\mathrm{Pd}\left(\mathrm{PPh}_{3}\right)_{4}(0.181 \mathrm{~g}$. $0.157 \mathrm{mmol})$ and $2 \mathrm{M}$ aq. $\mathrm{Na}_{2} \mathrm{CO}_{3}(6.0 \mathrm{~mL})$ by using toluene $(12.0 \mathrm{~mL})$ as solvent. The crude product was purified by recry'stallization from $n$-hexane to give $2 \mathbf{b}(1.78 \mathrm{~g} .78 \%)$ as a fluffy white solid: TLC $R_{f} 0.44\left(\mathrm{Et}_{2} \mathrm{O}: n\right.$-hexane $\left.=1: 4\right) ; \mathrm{mp}$ 76-77 ${ }^{\circ} \mathrm{C}:{ }^{1} \mathrm{H}$ NMR $\left(300 \mathrm{MHz} . \mathrm{CDCl}_{3}\right) \delta 0.90(\mathrm{~s}, 6 \mathrm{H}) .1 .38$ (s. $9 \mathrm{H}) .2 .57$ (s. $2 \mathrm{H}), 3.71$ (s. $2 \mathrm{H}) .6 .98-7.04$ (m. $2 \mathrm{H}) .7 .15-$ $7.23(\mathrm{~m}, 3 \mathrm{H}) .7 .53(\mathrm{~d}, J=8.56 \mathrm{~Hz}, 2 \mathrm{H}) .7 .59(\mathrm{~d} . J=8.56 \mathrm{~Hz}$. $2 \mathrm{H}) .7 .81$ (d. $J=8.73 \mathrm{~Hz}, 2 \mathrm{H}) .7 .98($ d. $J=8.73 \mathrm{~Hz}, 2 \mathrm{H}):{ }^{13} \mathrm{C}$ NMR (125 MHz. CDCl $\left.\mathrm{Cl}_{3}\right) \delta 24.2(\times 2) .31 .5(\times 3) .34 .9 .35 .5$, $44.4,77.5,126.3(\times 2), 126.5,127.2(\times 2), 127.8(\times 2), 128.1$ $(\times 2) .128 .6(\times 2) .130 .6(\times 2), 134.3 .136 .3,137.6,146.7$. 152.2: HRMS (EI. $70 \mathrm{eV}$ ) calcd for $\mathrm{C}_{27} \mathrm{H}_{32} \mathrm{O}_{3} \mathrm{~S}\left(\mathrm{M}^{-}\right)$ 436.2072, found 436.2071 . 
2,2-Dimethyl-3-phenyl-1-propyl $4^{\prime \prime}$-vinyl-1',1"-bijphenyl$4^{\prime}$-sulfonate (2c) was prepared by the reaction of $1 \mathrm{a}(2.00 \mathrm{~g}$. $5.22 \mathrm{mmol})$ with $p$-vinylphenylboronic acid $(0.85 \mathrm{~g}, 5.74$ mmol) in the presence of $\mathrm{Pd}\left(\mathrm{PPh}_{3}\right)_{4}(0.181 \mathrm{~g} .0 .157 \mathrm{numol})$ and $2 \mathrm{M}$ aq. $\mathrm{Na}_{2} \mathrm{CO}_{3}(6.0 \mathrm{~mL})$ by using toluene $(12.0 \mathrm{~mL})$ as solvent. The crude product was purified by recrystallization from $n$-hexane to give $2 \mathrm{c}(1.61 \mathrm{~g} .76 \%)$ as a slightly yellowish solid: TLC $R_{f} 0.53\left(\mathrm{Et}_{-} \mathrm{O}: n\right.$-hexane $\left.=1: 4\right): \mathrm{mp}$ $90-91{ }^{\circ} \mathrm{C} ;{ }^{1} \mathrm{H}$ NMR (300 MHz. CDCl 3$) \delta 0.90$ (s. $6 \mathrm{H}$ ), 2.57 (s. $2 \mathrm{H}) .3 .71(\mathrm{~s}, 2 \mathrm{H}) .5 .35(\mathrm{~d}, J=10.93 \mathrm{~Hz} .1 \mathrm{H}), 5.85$ (d. $J=$ $17.60 \mathrm{~Hz} .1 \mathrm{H}), 6.78(\mathrm{dd} . J=17.60,10.93 \mathrm{~Hz}, \mathrm{IH}), 7.00-7.03$ (m, 2H). $7.15-7.20(\mathrm{~m}, 3 \mathrm{H}) .7 .54(\mathrm{~d}, J=8.39 \mathrm{~Hz}, 2 \mathrm{H}) .7 .6 \mathrm{l}$ (d, $J=8.39 \mathrm{~Hz}, 2 \mathrm{H}), 7.78$ (d. $J=8.56 \mathrm{~Hz}, 2 \mathrm{H}) .7 .99$ (d. $J=$ $8.56 \mathrm{~Hz} .2 \mathrm{H}$ ): ${ }^{13} \mathrm{C}$ NMR $\left(75 \mathrm{MHz} \mathrm{CDCl}_{3}\right) \delta 24.1(\times 2), 35.4$. $44.4,77.4 .115 .3,126.6,127.2(\times 2), 127.8(\times 2) .127 .9(\times 2)$. $128.2(\times 2), 128.8(\times 2), 130.7(\times 2), 134.8,136.3,137.7$. 138.4. 138.6. 146.5: HRMS (EI. 70 eV) calcd for $\mathrm{C}_{25} \mathrm{H}_{2} \mathrm{O}_{3} \mathrm{~S}$ $\left(\mathrm{M}^{+}\right)$406.1603. found 406.1440.

2,2-Dimethyl-3-phenyl-1-propyl 1 ',1",1" 1 "terphenyl$4^{\prime}$-sulfonate (2d) was prepared by the reaction of $1 \mathrm{a}(2.00$ g. $5.22 \mathrm{mmol}$ ) with biphenylboronic acid (1.14 g. 5.74 mmol) in the presence of $\mathrm{Pd}\left(\mathrm{PPh}_{j}\right)_{4}(0.18 \mathrm{~g} .0 .157 \mathrm{mmol})$ and $2 \mathrm{M}$ aq. $\mathrm{Na}_{2} \mathrm{CO}_{3}(6.0 \mathrm{~mL})$ by using toluene $(12.0 \mathrm{~mL})$ as solvent. The crude product was purified by recrystallization from $n$-hexane to give $2 d(1.62 \mathrm{~g} .68 \%)$ as a fluffy white solid: TLC $R_{f} 0.50$ (UV $254 \mathrm{~mm}$ blue tailing spot. Et $\mathrm{t}_{-} \mathrm{O}: n$ hexane $=1: 1) ; \mathrm{mp} 161-162{ }^{\circ} \mathrm{C} ;{ }^{1} \mathrm{H}$ NMR $(500 \mathrm{MHz}$. $\left.\mathrm{CDCl}_{3}\right) \delta 0.90(\mathrm{~s}, 6 \mathrm{H}), 2.57$ (s. $\left.2 \mathrm{H}\right) .3 .72(\mathrm{~s}, 2 \mathrm{H}), 7.0 \mathrm{l}-7.03$ (m, $2 \mathrm{H}), 7.15-7.21$ (n. $3 \mathrm{H}), 7.39(\mathrm{t}, J=7.35 \mathrm{~Hz}, 1 \mathrm{H}), 7.48$ (dd. $J=7.80,7.35 \mathrm{~Hz}, 2 \mathrm{H}), 7.65(\mathrm{~d}, J=7.80 \mathrm{~Hz} .2 \mathrm{H}$ ), $7.7 \mathrm{l}$ (d, $J=8.70 \mathrm{~Hz}, 2 \mathrm{H}), 7.73$ (d. $J=8.70 \mathrm{~Hz}, 2 \mathrm{H}) .7 .82$ (d, $J=$ $8.25 \mathrm{~Hz}, 2 \mathrm{H}$ ), 8.00 (d. $J=8.25 \mathrm{~Hz} .2 \mathrm{H}) ;{ }^{13} \mathrm{C}$ NMR $(125$ $\left.\mathrm{MHz} . \mathrm{CDCl}_{\hat{j}}\right) \delta 24.2(\times 2) .35 .5,44.4 .77 .5,126.5 .127 .3$ $(\times 2), 127.6,127.8(\times 2), 127.9(\times 2), 128.0(\times 2), 128.1(\times 2)$. $128.7(\times 2), 129.1(\times 2), 130.6(\times 2), 134.7 .137 .5,138.0 .140 .4$. 141.8. 146.4; HRMS (EI, 70 eV) calcd for $\mathrm{C}_{29} \mathrm{H}_{28} \mathrm{O}_{3} \mathrm{~S}_{\left(\mathrm{M}^{+}\right)}$ 456.1756 . found 456.1766 .

2,2-Dimethyl-3-phenyl-1-propyl 4"-formyl-1',1"-bijphenyl$f^{\prime}$-sulfonate (2e) was prepared by the reaction of $1 \mathrm{a}(2.00 \mathrm{~g}$. $5.22 \mathrm{mmol}$ ) with $p$-formylphenylboronic acid $(0.86 \mathrm{~g} .5 .74$ mmol) in the presence of $\mathrm{Pd}\left(\mathrm{PPh}_{3}\right)_{4}(0.181 \mathrm{~g} .0 .157 \mathrm{nmmol})$ and $2 \mathrm{M}$ aq. $\mathrm{Na}_{2} \mathrm{CO}_{3}(6.0 \mathrm{~mL})$ by using toluene $(12.0 \mathrm{~mL})$ as solvent. The crude product was purified by recrystallization from $n$-hexane : $\mathrm{CH}_{2} \mathrm{Cl}_{2}(20: 1)$ to give $2 \mathrm{e}(1.37 \mathrm{~g}, 64 \%)$ as a pale yellowish solid: TLC $R_{f} 0.36\left(\mathrm{CH}_{2} \mathrm{Cl}_{2}\right)$; mp $122-123^{\circ} \mathrm{C}$ : ${ }^{1} \mathrm{H}$ NMR $\left(300 \mathrm{MHz} \mathrm{CDCl}_{j}\right) \delta 0.9 \mathrm{l}(\mathrm{s}, 6 \mathrm{H}) .2 .57$ (s. $\left.2 \mathrm{H}\right)$. 3.74 (s. 2H) $7.02-7.05$ (m. 2H) . 7.18-7.21 (m. 3H) . 7.79$7.83(\mathrm{~m}, 4 \mathrm{H}) .8 .01-8.06(\mathrm{~m}, 4 \mathrm{H}), 10.11(\mathrm{~s}, 1 \mathrm{H}):{ }^{13} \mathrm{C}$ NMR $\left(75 \mathrm{MHz}\right.$ acetone- $\left.d_{6}\right) \delta 24.2(\times 2), 35.9,44.7,78.3,127.3$. $129.0(\times 2), 129.2(\times 2), 129.5(\times 2), 129.8(\times 2), 131.3(\times 2)$. $131.5(\times 2) .136 .9,137.7,138.6,145.5,146.1,193.0$ : HRMS (EI. $70 \mathrm{eV}$ ) calcd for $\mathrm{C}_{24} \mathrm{H}_{24} \mathrm{O}_{4} \mathrm{~S}\left(\mathrm{M}^{-}\right) 408.1395$, found 408.1229 .

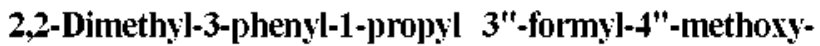
1',1"-biphenyl-4'-sulfonate (2f) was prepared by the reaction of $1 \mathrm{a}(2.00 \mathrm{~g} .5 .22 \mathrm{mmol})$ with 3-formyl-4methoxyphenylboronic acid (1.03 g. $5.74 \mathrm{mmol}$ ) in the presence of $\mathrm{Pd}\left(\mathrm{PPh}_{3}\right)_{4}(0.181 \mathrm{~g} .0 .157 \mathrm{nmol})$ and $2 \mathrm{M}$ aq. $\mathrm{Na}_{2} \mathrm{CO}_{3}(6.0 \mathrm{~mL})$ by using toluene $(12.0 \mathrm{~mL})$ as solvent. The crude product was purified by recrystallization from $n$ hexane : $\mathrm{CH}_{2} \mathrm{Cl}_{2}(20: 1)$ to give $2 \mathrm{f}(1.69 \mathrm{~g} .74 \%)$ as a bright canary yellow solid: $\operatorname{mp} 83-84{ }^{\circ} \mathrm{C}$; TLC $R_{f} 0.38\left(\mathrm{CH}_{2} \mathrm{Cl}_{2}\right):{ }^{1} \mathrm{H}$ NMR (300 MHz, CDCl $) \delta 0.89(\mathrm{~s}, 6 \mathrm{H}), 2.57$ (s. 2H). 3.71 (s, 2H). 4.02 (s. 3H), 7.00-7.06 (m, 2H). 7.17-7.23 (m. 3H), 7.14 (d. $J=8.73 \mathrm{~Hz}, 1 \mathrm{H}$ ). 7.76 (d. $J=8.73 \mathrm{~Hz}, 2 \mathrm{H}$ ). 7.85 (dd. $J=8.73,2.52 \mathrm{~Hz}, 1 \mathrm{H}$ ). $7.98(\mathrm{~d}, J=8.73 \mathrm{~Hz}, 2 \mathrm{H}$ ). 8.13 (d, $J=2.52 \mathrm{~Hz}, 1 \mathrm{H}), 10.54(\mathrm{~s}, 1 \mathrm{H}):{ }^{12} \mathrm{C} \mathrm{NMR}(75 \mathrm{MHz}$, $\left.\mathrm{CDCl}_{3}\right) \delta 24.1(\times 2) .35 .4,44.3 .56 .1,77.5 .112 .8,125.3$, 126.5. 127.5 (×2), 128.2(×2), $128.9(\times 2) .130 .7(\times 2) .131 .7$, 132.3, 132.4. 134.7, 137.6, 145.2, 162.5. 189.7. HRMS (EI. $70 \mathrm{eV}$ ) calcd for $\mathrm{C}_{25} \mathrm{H}_{26} \mathrm{O}_{4} \mathrm{~S}\left(\mathrm{M}^{+}\right) 438.1501$, found 438.1548

Neopentyl +'-formyl-1,1'-biphenyl-3-sulfonate (2g) was prepared by the reaction of $1 \mathrm{~b}(0.40 \mathrm{~g}, 1.30 \mathrm{~mm}$ ol) with 4 formylphenylboronic acid $(0.21 \mathrm{~g} .1 .43 \mathrm{mmol})$ in the presence of $\mathrm{Pd}\left(\mathrm{PPh}_{3}\right)_{4}(0.045 \mathrm{~g}, 0.039 \mathrm{mmol})$ and $2 \mathrm{M}$ aq $\mathrm{Na}_{2} \mathrm{CO}_{3}(1.5 \mathrm{~mL})$ by using toluene $(10.0 \mathrm{~mL})$ as solvent. The crude product was purified by column chromatography to give $2 \mathrm{~g}(0.30 \mathrm{~g}, 69 \%)$ as a pale ivorish solid: TLC $R_{f} 0.31$ $\left(\mathrm{CH}_{2} \mathrm{Cl}_{2}\right)$ : mp 68-70 ${ }^{\circ} \mathrm{C}:{ }^{1} \mathrm{H}$ NMR $\left(500 \mathrm{MHz} . \mathrm{CDCl}_{3}\right) \delta 0.92$ (s, 9H). 3.74 (s. $2 \mathrm{H}), 7.68$ (t. $J=7.80 \mathrm{~Hz}, 1 \mathrm{H}) .7 .78$ (d. $J=$ $8.35 \mathrm{~Hz}, 2 \mathrm{H}$ ). 7.90 (ddd, $J=7.80 .1 .85,0.90 \mathrm{~Hz} .1 \mathrm{H}) .7 .94$ (ddd, $J=7.80 .1 .85 .0 .90 \mathrm{~Hz}, \mathrm{lH}) .8 .00(\mathrm{~d}, J=8.35 \mathrm{~Hz} .2 \mathrm{H}$ ), 8.16 (t. $J=1.85 \mathrm{~Hz} .1 \mathrm{H}), 10.08(\mathrm{~s} .1 \mathrm{H}) ;{ }^{13} \mathrm{C}$ NMR (125 $\left.\mathrm{MHz}, \mathrm{CDCl}_{3}\right) \delta 26.2(\times 3) .31 .9 .80 .1,126.7 .127 .7,128.0$ $(\times 2) .130 .1,130.7(\times 2), 132.5,136.2 .137 .4,141.3 .144 .9$; HRMS (EI. $70 \mathrm{eV}$ ) calcd for $\mathrm{C}_{18} \mathrm{H}_{21} \mathrm{O}_{4} \mathrm{~S}\left(\mathrm{M}^{-}\right) 332.1082$, found 332.1092 .

Neopentyl 4'-formyl-1,1'-bijhenyl-2-sulfonate (2h) was prepared by the reaction of $1 \mathrm{c}(0.50 \mathrm{~g} .1 .63 \mathrm{mmol})$ with $p$ formylphenylboronic acid $(0.27 \mathrm{~g} .1 .79 \mathrm{mmol})$ in the presence of $\mathrm{Pd}\left(\mathrm{PPh}_{3}\right)_{4}(0.057 \mathrm{~g}, 0.049 \mathrm{mmol})$ and $2 \mathrm{M}$ aq. $\mathrm{Na}_{2} \mathrm{CO}_{3}(2.0 \mathrm{~mL})$ by using toluene $(10.0 \mathrm{~mL})$ as solvent. The crude product was purified by column chromatography to give $2 \mathrm{~h}(0.36 \mathrm{~g}, 66 \%)$ as a viscous colorless oil: TLC $R_{f} 0.31$ $\left(\mathrm{CH}_{2} \mathrm{Cl}_{2}\right):{ }^{1} \mathrm{H}$ NMR $\left(500 \mathrm{MHz}, \mathrm{CDCl}_{3}\right) \delta 0.82(\mathrm{~s}, 9 \mathrm{H}) .3 .59$ (s. $2 \mathrm{H}) .7 .39(\mathrm{dd} . J=7.78 .0 .90 \mathrm{~Hz}, 1 \mathrm{H}) .7 .58(\mathrm{td} . J=7.78$ $0.90 \mathrm{~Hz}, 1 \mathrm{H}), 7.60(\mathrm{~d}, J=8.25 \mathrm{~Hz} .2 \mathrm{H}) .7 .68(\mathrm{td} . J=7.80$ $0.90 \mathrm{~Hz}, 1 \mathrm{H}) .7 .93(\mathrm{dd} . J=8.25 \mathrm{~Hz}, 2 \mathrm{H}) .8 .13(\mathrm{dd} . J=7.80$. $0.90 \mathrm{~Hz}, 1 \mathrm{H}), 10.07$ (s. $1 \mathrm{H}):{ }^{13} \mathrm{C} \mathrm{NMR}\left(125 \mathrm{MHz}^{\mathrm{C}} \mathrm{CDCl}_{3}\right) \delta$ $26.2(\times 3), 31.8 .79 .7,128.6,129.2(\times 2) .130 .0 .130 .3(\times 2)$, $132.5,133.4,135.2,136.0,140.8,145.4 .192 .1$ : HRMS (EI. $70 \mathrm{eV}$ ) calcd for $\mathrm{C}_{18} \mathrm{H}_{2 j} \mathrm{O}_{4} \mathrm{~S}\left(\mathrm{M}^{+}\right)$332.1082, found 332.1059

\section{References}

1. (a) Hassan. T.: Sévignon1. M.: Gozzi. C.: Schulz. E.: Lemaire. M Chent Rer: 2002. 102. 1359. (b) Litthe. A. F.: Fu. G. C. Angew: Chem. Iht Ed. 2002. 11,4176 . (c) Stanforth. S. P. Tetrahedron 1998. 54. 263. (d) Tsuji. J. Palladium Reagents and Catalysts. Imovations in Organic Symhesis: Wiley: New York, 1995.

2. (a) Suzuki. A. J. Organonet Chent 1999.576. 147. (b) Myaura N.: Suzuki. A. Chem. Rev 1995. 95. 2457. (c) Kim. Y. H.: Aht1. J H.: Shin. D. C.: Kim. J. H.: Park. Y. W.: Choi. D. S.: Kim. Y. K.: Kwon. S. K. Bull Korean Chem. Soc. 2001. 22. 1181 . (d) Paek, K. Bull. Korean Chem. Soc, $1991,12,599$

3. Stille, J. K. Angew. Chem. Int Ed Engl. 1986, 25, 508. 
4. (a) Erdik. E. In Organozinc Reagemts in Organic Symthesis: CRC Press. 1996: pp 271. (b) Quesnelle. C. A.: Familoni. O. B.: Snieckus. V. Sinlent 1994. 349. (c) Park. K.: Yuan. K.: Scott. W. J. J. Org Chem 1993, 58, 4866. (d) Negishi. E. Acc. Chem. Res. $1982,15,340$.

5. (a) Ylan. K.: Scott, W. I. Tetrahedron Lett. 1991, 32, 189. (b) Hayashi. T.: Konjishi. M.: Yokota. K.: Kumada. M. J. Organomet. Chem 1985 285. 359. (c) Hayashi. T.: Konishi. M.: Kobori. Y: Kumada. M.: Higuchi. T.: Hirotsu. K. J. Am. Chem. Soc. 1984. 106, 158. (d) Tamao, K.: Sumitani. K.: Kiso, Y.: Zembayashi, M.: Fujioka, A.: Kodama, S.: Nakajima, I.: Minato. A.: Kumada, M. Buhl. Chem. Soc. Jpn. 1976, 49. 1958.

6. (a) Hallock. Y. F.: Manfredi. K. P.: Blunt. J. W.: Cardellina. J. H.. II: Schäffer. M.: Gulden. K.-P.: Bringmann. G.: Lee. A. Y: Clardy. J.: François. G.: Bovd. M. R. J. Org Chem. 1994. 59. 6349. (b) Chang, L. L.; Ashton, W. T:; Flanagan. K. L.: Strelitz, R. A.: Maccoss, M.: Greenlee, W. I.: Chang. R. S. L.: Lotti. V. J: Faust. K. A.: Chen, T. B.: Bunting, P. Zingaro, G. J.: Kitlighn. S. D.: Siegl. P. K. S. J. hed Chem. 1993. 36. 2558. (c) Bovy. P. R.: Collins. J. T.: Olins. G. M.: McMahon. E. G.: Hutton. W. C. $J$. Hed Chem 1991.34.2410.

7. (a) Hohnholz. D.: Schweikart. K.-H.: Subramanian, L. R: Wedel, A.: Wischert W: Hanack. M. Symth. Met. 2000. 110. 141. (b) Miyashita, A.; Karino. H.: Slimamura, J.: Chiba. T; Nagano, K.: Nohira. H.: Takaya. H. Chem Lett 1989. 1849. (c) Combellas. C.: Gautier. H.: Simon. J: Thiebault. A.: Tournilhac. F.: Barzoukas. M.: Josse. D.: Ledoux. I.: Amatore. C.: Verpeaux. J.-N. J. Chem. Soc. Chem. Commm. 1988. 203. (d) Solladie. G.: Gottarelli. G. Tetratiedron 1987, 43.1425 .

8. Mvaura. N.: Yanagi. T.: Suzuki. A. Symh Gommm. 1981. 11.513.

9. (a) Pourbaix. C.: Carreaux. F.: Carboni. B. Org. Lett. 2001. 3. 803. (b) Chamoin. S.: Houldsworth. S.: Kruse. C. G.: Bakiker. W. I.: Stiechus. V. Tetrahedron Lett. 1998. 39. 4179. (c) Ruhland. B.: Bombrut1. A.: Gallop. M. A. J. Org. Chent 1997. 62. 7820. (d) Guiles. J. W.; Johnson. S. G.; Murray, W. V. J. Org. Chem. 1996. 61. 5169 . (e) Larhed. M.: Lindeberg, G.: Hallberg. A. Tetrahedron Lett. 1996, 37, 8219. (t) Backes. B. J.; Ellman. J. A. J. Am. Chem. Soc. 1994. 116. 11171 . (g) Frenette. R.: Friesen. R. W. Tetrahedon Lett. 1994. 35.9177.

10. Casalnuovo. A. L.: Calabrese. J. C. J. Am. Chent Soc. 1990.112. 4324 .

11. Chan. M. F; Kois, A.: Verner, E. J; Raju. B. G.: Castillo, R. S. Wu. C.: Okun. I.: Stavros. F. D.; Balaji, V. N. Bioorg Med Chem. 1998. 6.2301.

12. For the nickel-catalyzed coupling reaction of alhyloxysulfonylarenes via the displacement of alkyloxysulfonyl groups see: Cho. C.-H.: Yun. H.-S.; Park. K. J. Org. Chem. 2003, 68. 3017.

13. For the transition metal-catalyzed coupling reaction of organic tosylates via the displacement of tosylates see: (a) Zim, D.; Lando. V. R.: Dupont. T.: Monteiro. A. L. Org. Lett. 2001. 3. 3049. (b) Nagatsugi. F.: Uemura. K.: Nahashima. S.: Maeda. M.: Sasaki. S. Tetrohedron 1997. 53. 3035. (c) Nagatsugi. F.: Uemura. K.: Nakashima. S.; Maeda. M.; Sasaki. S. Tetrahedron Lett. 1995. 36. 421

14. (a) Feuerstein, M; Doucet. H.: Santelli, M. Synlett 2001. 1458. (b) Inada. K.: Miyaura. N. Tetrahadron 2000. 56. 8661. (c) Wolfe. J. P.: Singer. R. A.: Yang. B. H.: Buchwald. S. L. J. Am. Chem. Soc 1999. 121.9550. (d) Wolte. J. P.: Buchwald. S. L. Angew: Chen. Int Ed. 1999, 38. 2413. (e) Shen. W. Tetrahedhon Lelt. 1997, 38. 5575 .

15. Diorazio, L. J.: Widdowson. D. A.: Clough. J. M. Tetrahedron 1992. 48. 8073 . 